\title{
EMPREGO DE DIAGRAMAS DE FASES PSEUDO- TERNÁRIOS PARA PREPARAÇÃO DE SISTEMAS CONTENDO ELAVADO TEOR DE EXTRATOS VEGETAIS
}

\author{
K. M. T. SILVA ${ }^{1}$; I. C. F. BEZERRA ${ }^{2}$; M. R. A. FERREIRA ${ }^{1,2}$ L. A. L. SOARES ${ }^{1,2}$ \\ ${ }^{1}$ Universidade Federal de Pernambuco, Centro de Ciências da Saúde, Departamento de Ciências \\ Farmacêuticas \\ ${ }^{2}$ Programa de Pós-graduação em Inovação Terapêutica, Universidade Federal de Pernambuco, Centro \\ de Ciências Biológicas \\ E-mail para contato: kassiateixeira95@hotmail.com \\ RESUMO - Diagramas de fases pseudo-ternários consistem em uma das formas mais \\ eficientes para o desenvolvimento e preparação de sistemas emulsionados, esta \\ ferramenta permite a identificação das diferentes regiões de formação de sistemas, além \\ de apontar a melhor combinação e proporção de ingredientes da formulação. Este \\ trabalho visa à construção de diagramas de fase pseudo-ternários a fim de obter \\ formulações para veiculação de extratos vegetais. Os diagramas de fases pseudo- \\ ternários foram construídos por dois métodos (ultraturrax e sonda ultrassônica), utilizado \\ como fase oleosa Miglyol $812^{\circledR}$ e Span ${ }^{\circledR}$ 80; e como fase aquosa, água destilada e Tween ${ }^{\circledR}$ \\ 20. Em cada diagrama construido, foram obtidas 81 formulações. Os resultados \\ indicaram que os sistemas preparados por sonda ultrassônica foram os que apresentaram \\ sistemas com melhores propriedades tecnológicas. O emprego de diagramas pseudo- \\ ternários resultaram em desenvolvimento racional dos sistemas, de maneira simples e \\ econômica.
}

Palavras chave: Sistemas dispersos. Emulsão. Diagrama de fases.

ABSTRACT - Pseudo-ternary phase diagrams consist of one of the most efficient ways for the development and manufacture of emulsified systems, this tool allows to identify the different regions of systems formation as well to point out the one that represents the better combination and proportion of formulation ingredients. This aims of this work was the use of pseudo-ternary phase diagrams to produce disperse systems for carrying herbal extracts. The pseudo-ternary phase diagrams were prepared by using two methods (ultraturrax and ultrasonic), and the ingredients were: Miglyol $812^{\circledR}$ and Span ${ }^{\circledR}$ 80, as oil

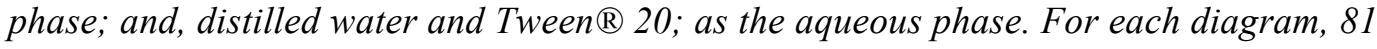
formulations were prepared. The results indicated that the systems manufactured by ultrasonic homogenizer showed better technological properties. The use of pseudo-ternary diagrams as manufacture strategy resulted in a rational and simple development approach of the systems.

Keywords: Dispersed systems. Emulsion. Phases diagram. 


\section{INTRODUÇÃO}

As emulsões são sistemas dispersos estáveis formados pela presença de tensoativos e dois ou mais líquido imiscíveis, onde, um se caracteriza como, fase dispersa, e outro como fase dispersante (MCCLEMENTS, 2011). Podem ser classificadas de acordo com a natureza da fase dispersante, caracterizando-se como O/A (óleo em água) quando as gotículas de óleo estão dispersas em água; A/O (água em óleo) quando a água encontra-se dispersa em óleo; e, O/A/O (óleo-água-óleo) ou $\mathrm{A} / \mathrm{O} / \mathrm{A}$ (água-óleo-água) quando se trata de preparações múltiplas (LACHMAN; LIEBERMAN; KANIG, 2015; AULTON, 2016). Tais formulações apresentam elevado potencial como sistemas de liberação, devido a sua capacidade de solubilizar tanto substâncias hidrofílicas em meio lipofílico, quanto lipofílicas em meio aquoso e anfifílico na interface óleo/água (KE et al., 2005; OLIVEIRA et al., 2004; SILVA et al., 2009). O sistema de equilíbrio das emulsões podem ser representados através de diagrama de fases pseudo-ternário, onde cada vértice do triângulo representa $100 \%$ de dado componente em particular. O diagrama permite identificar as diferentes regiões formadas bem como apontar aquela que representa a melhor combinação dos componentes da formulação e suas respectivas quantidades (SILVA et al., 2009).

Nesse contexto, o trabalho teve como objetivo construir diagramas de fase pseudoternários, e analisar a reprodutibilidade das formulações relativas às regiões delimitadas do diagrama, para a obtenção de formulações promissoras visando a incorporação de extratos vegetais.

\section{MATERIAIS E MÉTODOS}

\subsection{Construção de Diagramas de fases}

Os diagramas de fase pseudo-ternários foram construídos a partir de dois métodos de obtenção (ultraturrax - IKA ${ }^{\circledR}$ e sonda ultrassônica - Ultronique ${ }^{\circledR}$ ), utilizadando como fase oleosa Miglyol $812^{\circledR}$ e $\operatorname{Span}^{\circledR} 80$, e como fase aquosa a água destilada e Tween ${ }^{\circledR} 20$.

As dispersões foram preparadas com diferentes proporções óleo/tensoativo, seguindo a razão 1:9, 2:8, 3:7, 4:6, 5:5, 6:4, 7:3, 8:2 e 9:1, obtendo 9 formulações a cada proporção, utilizado de titulações crescentes com água destilada. Para ambas as metodologias o ciclo de obtenção foi o mesmo, sendo 1 minuto sob agitação mecânica e 3 minutos em banho de ultrassom. $O$ procedimento foi realizado duas vezes, com repouso de 5 minutos entre os ciclos, para realização de análise e caracterização visual.

\subsection{Análise do diagrama de fases e reprodução das formulações}

As formulações obtidas foram caracterizadas visualmente em diferentes sistemas de acordo com o comportamento das fases, sendo classificadas em: separação de fases, microemulsão, nanoemulsão, emulsão, emulsão viscosa, emulsão líquida e creme. Após a preparação e caracterização de todas as formulações, os diagramas foram determinados com o auxílio do software Origin ${ }^{\circledR}$. Após obtenção dos diagramas de fases, foram preparadas emulsões para reprodução do comportamento das fases. Estas foram preparadas através do método de inversão de fases, que consiste na dispersão dos tensoativos na fase aquosa (Tween $20^{\circledR}$ ) e oleosa $\left(\right.$ Span $80^{\circledR}$ ), separadamente, seguido do aquecimento de ambas as fases a $70^{\circ} \mathrm{C}$, em banho maria (Lucadema $^{\circledR}$ ). Após aquecimento, as fases foram misturadas e homogeneizadas sob ação mecânica em sonda ultrassônica/ultraturrax por 10 minutos. As emulsões obtidas foram analisadas segundo $\mathrm{pH}$ e características macroscópicas (monitoradas durante 15 dias). 


\section{RESULTADOS E DISCUSSÃO}

Em cada diagrama construído, foram obtidas 81 formulações, e de acordo com a análise visual, foi possível evidenciar regiões de separação de fases, microemulsões, nanoemulsões, cremes e três diferentes tipos de emulsões: viscosas, intermediárias e líquidas (Figura 1). Os diagramas possibilitaram a identificação de regiões de emulsões em ambos os métodos de dispersão, porém, as emulsões obtidas por sonda ultrassônica apresentaram regiões bem delimitadas e melhores características macroscópicas. Estas características podem ser provenientes do menor tamanho de gotículas comumente apresentado por preparações obtidas por sondas ultrassônicas. Isto ocorre pela capacidade do equipamento de gerar ondas ultrassônicas muito intensas e uniformes que são capazes de romper e misturar as fases oleosas e aquosas, onde o tamanho das gotículas formadas é inversamente proporcional a potência e intensidade da onda ultrassônica. O que explica a presença de boas características pelos sistemas obtidos, visto que a potência utilizada para a preparação dos sistemas foi de $90 \%$.

Partindo do diagrama de fases realizado com a sonda utrassônica (Figura 1-B), foram selecionadas e reproduzidas às formulações com regiões bem definidas a fim de garantir a reprodutibilidade do diagrama de fases em questão, estas estão representadas na tabela 2. Dentre as formulações obtidas apenas as F5 e F6 não apresentaram reprodutibilidade adequada, apresentando separação de fases. As demais formulações apresentaram $\mathrm{pH}$ ácido, compatíveis com o $\mathrm{pH}$ da pele. Considerando as características macroscópicas, apresentaram-se como emulsões brancas, líquidas, uniformes e ausentes de creme desde o momento de sua obtenção até o final dos 15 dias de monitoramento, evidenciando a reprodução do comportamento das fases e indicando que estes sistemas são promissores para incorporação de extratos vegetais, necessitando de novos ensaios de caracterização e estabilidade.

Figura 1- Diagramas de fases pseudo-ternários obtidos por ultra-turrax (A) e sonda ultrassônica (B).
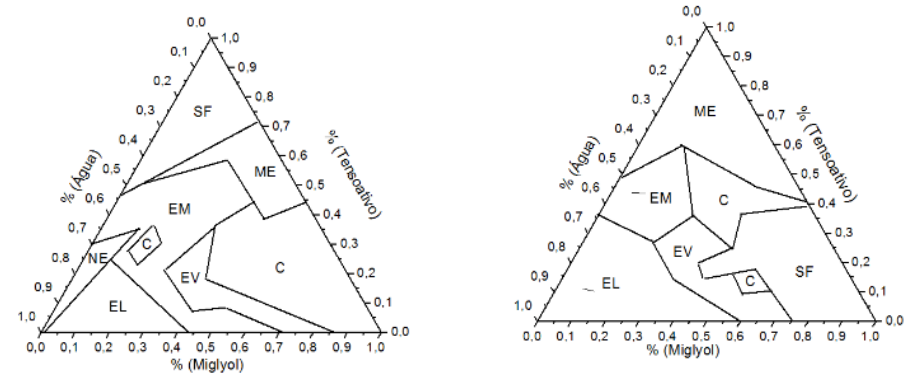

Tabela 2- Proporção dos constituintes das formulações selecionadas a partir do diagrama de fases obtido por sonda ultrassônica.

\begin{tabular}{lcccc}
\hline Form. & $\begin{array}{c}\text { Água } \\
(\mathbf{\% )}\end{array}$ & $\begin{array}{c}\text { Óleo } \\
\mathbf{( \% )}\end{array}$ & $\begin{array}{c}\text { Tensioativo } \\
(\mathbf{\%})\end{array}$ & $\mathbf{p H}$ \\
\hline F1 & 88,0 & 10,0 & 2,0 & 4,15 \\
F2 & 75,0 & 25,0 & 3,0 & 4,49 \\
F3 & 93,0 & 5,0 & 2,0 & 4,68 \\
F4 & 92,0 & 5,0 & 3,0 & 4,35 \\
F5 & 93,5 & 5,0 & 1,5 & - \\
F6 & 95,0 & 3,0 & 2,0 & - \\
F7 & 40,0 & 36,0 & 24,0 & 4,22 \\
\hline
\end{tabular}




\section{CONCLUSÃO}

\section{ENCONTRO BRASILEIRO PARA INOVAÇÃO TERAPÊUTICA}

A construção de diagrama pseudo-ternário trata-se de uma técnica simples, que possibilitou a identificação das diversas regiões obtidas facilitando a escolha da melhor combinação dos componentes das formulações em suas respectivas quantidades a fim de produzir um sistema mais estável. Além disso, a melhor forma para a obtenção das formulações é por sonda ultrassônica devido às suas regiões mais definidas decorrente do menor tamanho de gotícula gerado. Além disso, foi evidenciada a reprodução das regiões apresentadas no diagrama, as quais obtiveram estabilidade satisfatória durante o tempo de monitoramento.

\section{REFERÊNCIAS}

AULTON, M. E. Delineamento de Formas Farmacêuticas. 4 ed. Porto Alegre: Elsevier, 2016. 872p.

LACHMAn, L.; LIEBERMAN, H. A.; KANIG, J. L. Teoria e prática na indústria farmacêutica. 3. Ed. Lisboa: Fundação Calouste Gulbenkian, 2015. v. 2. 1517 p.

MCCLEMENTS, D. J. Edible nanoemulsions: fabrication, properties, and functional performance. The Roy. Soc. Chemis., v. 7, p. 2297-2316, 2011.

SILVA, J. A.; DAMASCENO, B. P. G. L.; BORBA, V. F. C.; EGITO, E. S. T.; SANTANA, D. P. Uso de diagramas de fase pseudoternários como ferramenta de obtenção de nanoemulsões transdérmicas. Rev. Bras. Farm., v. 90, n. 3, p. 245-249, 2009.

OLIVEIRA, A. G.; SCARPA, M. V.; CORREA, M. A.; CERA, L. R. \& FORMARIZ, T. P. Microemulsões: estrutura e aplicações como sistema de liberação de fármacos. Quím. Nova, v. 27, n. 1, p. 131-138, 2004.

\section{AGRADECIMENTOS}

Os autores agradecem ao PIBIC-UFPE, FACEPE (IBPG-0557-4.03/15; APQ-0493- 4.03/14) e ao CNPq (308386/2015-9). 